International Mathematical Forum, 2, 2007, no. 49, 2447 - 2452

\title{
Pairwise Semi Paracompact Spaces
}

\author{
Jamal M. Mustafa \\ Department of Mathematics \\ Al al-Bayt University \\ P.O. Box: 130095 \\ Mafraq, Jordan \\ jjmmrr971@yahoo.com
}

\begin{abstract}
The purpose of the present paper is to study the concept of pairwise semi paracompactness in bitopological spaces. We give some characterizations of pairwise semi-paracompact spaces. We shall introduce two types of pairwise semi-paracompact spaces and then discuss the relation between them.
\end{abstract}

Mathematics Subject Classification: 54E55, 54D20

Keywords: Pairwise-semi-paracompact spaces, $p-$ semi-regular spaces, $p-$ semi-normal spaces, $p-$ semi-compact spaces

\section{Introduction.}

The concept of bitopological spaces was initiated by Kelly [6]. A set $X$ equipped with two topologies $\tau_{1}$ and $\tau_{2}$ is called a bitopological space denoted by $\left(X, \tau_{1}, \tau_{2}\right)$. Semi - open sets were studied by Levine [7], Crosseley [1], [2], Dorsett [3], [4] and Hanna and Dorsett [5].

Let $(X, \tau)$ be a topological space and $A$ be a subset of $X$. We denote the closure of $A$ ( the interior of $A$ respectively) by $C l(A)$ ( $\operatorname{Int}(A)$ respectively ).

A subset $A$ of $(X, \tau)$ is called semi - open [7] if $A \subseteq C l(\operatorname{Int}(A))$. The complement of a semi-open set is called semi-closed [1]. The family of all semi - open sets in $(X, \tau)$ is denoted by $S(\tau)$.

Let $A$ be a subset of a bitopological space $\left(X, \tau_{1}, \tau_{2}\right)$. The closure of $A$ and the interior of $A$ with respect to $\tau_{i}$ are denoted by $C l_{i}(A)$ and $\operatorname{Int}_{i}(A)$ respectively. 
The intersection of all semi-closed sets containing $A$ is called the semiclosure [1] of $A$, denoted by $\operatorname{sCl}(A)$. The semi-closure of $A$ with respect to $S\left(\tau_{i}\right)$ is denoted by $s C l_{i}(A)$.

\section{Pairwise Semi Paracompact spaces.}

In this section we define and study two types of pairwise semi-paracompact spaces namely $p_{1}-$ sem $i$-paracompact and $p_{2}-$ semi-paracompact spaces.

Definition 2.1. A family $\tilde{A}$ of subsets of a bitopological space $\left(X, \tau_{1}, \tau_{2}\right)$ is called $\tau_{1} \tau_{2}-$ semi-open if $\tilde{A} \subset S\left(\tau_{1}\right) \cup S\left(\tau_{2}\right)$. If, in addition $\tilde{A} \cap S\left(\tau_{1}\right) \neq \phi$ and $\tilde{A} \cap S\left(\tau_{2}\right) \neq \phi$ then $\tilde{A}$ is called pairwise semi - open ( simply $p-$ semi - open ) .

Definition 2.2. If $\tilde{U}$ and $\tilde{N}$ are $\tau_{1} \tau_{2}-$ semi-open covers of the bitopological space $\left(X, \tau_{1}, \tau_{2}\right)$, then $\tilde{U}$ is called a refinement of $\tilde{N}$ if each $U \in \tilde{U} \cap S\left(\tau_{i}\right)$ is contained in some $V \in \tilde{N} \cap S\left(\tau_{i}\right), i=1,2$.

Definition 2.3. A family $\tilde{A}$ of subsets of a space $(X, \tau)$ is locally finite in $(X, S(\tau))$ if for each $x \in X$ there exists a semi - open set $U$ such that $x \in U$ and $U$ intersects at most finitely many elements of $\tilde{A}$.

Definition 2.4. A collection $\tilde{A}$ of subsets of the bitopological space $\left(X, \tau_{1}, \tau_{2}\right)$ is called $p_{1}$-locally finite if $\tilde{A} \cap S\left(\tau_{i}\right)$ is locally finite in $\left(X, S\left(\tau_{i}\right)\right), i=$ 1,2 .

Definition 2.5. A collection $\tilde{A}$ of subsets of the bitopological space $\left(X, \tau_{1}, \tau_{2}\right)$ is called $p_{2}$-locally finite if $\tilde{A} \cap S\left(\tau_{i}\right)$ is locally finite in $\left(X, S\left(\tau_{j}\right)\right), i \neq$ $j ; i, j=1,2$.

Definition 2.6. A bitopological space $\left(X, \tau_{1}, \tau_{2}\right)$ is called $p_{2}-$ semiparacompact ( $p_{1}-$ sem $i-$ paracompact respectively $)$ if each $p-s e m i-$ open cover of $X$ has a $p_{2}$-locally $\left(p_{1}\right.$-locally respectively $)$ finite $\tau_{1} \tau_{2}-$ semi-open refinement.

Notice that $p_{1}-$ semi-paracompactness does not imply $p_{2}-$ semiparacompactness and $p_{2}-s e m i-$ paracompactness does not imply $p_{1}-$ semiparacompactness as shown in the following two examples: 
Example 2.7. Let $X=R$ and $\tau_{1}, \tau_{2}$ be the topologies on $X$ generated by the bases $\beta_{1}, \beta_{2}$ respectively, defined by

$\beta_{1}=\{\{x\}: x \geq 0\} \cup\{(-\infty, 0)-F: F$ is any finite set $\}$, and

$ß_{1}=\{\{x\}: x \prec 0\} \cup\{[0, \infty)-F: F$ is any finite set $\}$.

Then $\left(X, \tau_{1}, \tau_{2}\right)$ is $p_{1}-$ sem $i$-paracompact space but not $p_{2}-$ semi-paracompact for the $p-$ semi-open cover $\{\{x\}: x \in X\}$ has no $p_{2}$-locally finite $\tau_{1} \tau_{2}-$ semi - open refinement.

Example 2.8. Let $X=R$ and $\tau_{1}, \tau_{2}$ be the topologies on $X$ generated by the bases $\beta_{1}, \beta_{2}$ respectively, defined by

$\beta_{1}=\{\{x\}: x \leq 0\} \cup\{\{y\} \cup(-\infty, 0)-F: y \succ 0$ and $F$ is any finite set $\}$, and

$\beta_{1}=\{\{x\}: x \succ 0\} \cup\{[0, z): z \succ 0\} \cup\{\{y\} \cup(0, \infty)-F: y \prec 0$ and $F$ is any finite set $\}$.

Then $\left(X, \tau_{1}, \tau_{2}\right)$ is $p_{2}-$ sem $i$-paracompact space but not $p_{1}-$ semi-paracompact for the $p-$ semi-open cover $\{\{x\}: x \in X\}$ has no $p_{1}$-locally finite $\tau_{1} \tau_{2}-$ semi - open refinement.

Definition 2.9. A subset $F$ of a space $(X, \tau)$ is called $\tau$-semi-paracompact relative to $X$ if every semi-open cover of $F$ by members of $S(\tau)$ has a locally finite ( in $X$ ) refinement by members of $S(\tau)$.

Theorem 2.10. For the bitopological space $\left(X, \tau_{1}, \tau_{2}\right)$ the following are equivalent:

i) $\left(X, \tau_{1}, \tau_{2}\right)$ is $p_{1}-$ semi-paracompact.

ii) Each $\tau_{1}-$ semi-closed proper subset of $X$ is $\tau_{2}-$ semi-paracompact relative to $X$, and each $\tau_{2}-$ semi-closed proper subset of $X$ is $\tau_{1}-$ semi-paracompact relative to $X$.

Proof. $(i) \longrightarrow(i i)$. Let $F \neq \phi$ and $F \neq X$ be a $\tau_{1}-$ semi - closed subset of $X$ and $\tilde{U}=\left\{U_{\alpha}: \alpha \in \Delta\right\}$ be a $\tau_{2}-$ semi-open cover of $F$. Then $\tilde{N}=\left\{U_{\alpha}: \alpha \in \Delta\right\} \cup\{X-F\}$ is a $p-$ semi-open cover of $X$. But $\left(X, \tau_{1}, \tau_{2}\right)$ is $p_{1}-$ semi-paracompact, then $\tilde{N}$ has a $p_{1}$-locally finite $\tau_{1} \tau_{2}-$ semi-open refinement, say $\left\{U_{\alpha}^{\prime}: \alpha \in \Delta\right\} \cup\left\{V_{\beta}: \beta \in \Gamma\right\}$, where $U_{\alpha}^{\prime} \in S\left(\tau_{2}\right)$ and $V_{\beta} \in S\left(\tau_{1}\right)$ for each $\alpha \in \Delta$ and $\beta \in \Gamma$. Thus $\left\{U_{\alpha}^{\prime}: \alpha \in \Delta\right\}$ is a $S\left(\tau_{2}\right)$-locally finite $\tau_{2}$ - semi-open refinement of $\tilde{U}$ for $F$. Hence $F$ is $\tau_{2}-$ semi-paracompact relative to $X$. Similarly each $\tau_{2}-$ semi - closed proper subset of $X$ is $\tau_{1}-$ semi-paracompact relative to $X$.

$(i i) \longrightarrow(i)$. Let $\tilde{U}$ be a $p-$ semi - open cover of $\mathrm{X}$, say $\tilde{U}=\left\{U_{\beta}: \beta \in\right.$ $\Gamma\} \cup\left\{V_{\alpha}: \alpha \in \Delta\right\}$ where $U_{\beta} \in S\left(\tau_{1}\right)$ and $V_{\alpha} \in S\left(\tau_{2}\right)$ for all $\beta \in \Gamma$ and all $\alpha \in \Delta$. Now we have two cases: 
Case 1: If $\cup\left\{V_{\alpha}: \alpha \in \Delta\right\}=X$, choose $\beta_{0} \in \Gamma$ such that $U_{\beta_{0}} \neq \phi$. Since $\left\{V_{\alpha}: \alpha \in \Delta\right\}$ is a $\tau_{2}-$ semi-open cover of the $\tau_{1}-$ semi-closed proper subset $X-U_{\beta_{0}}$, therefore it has a $\tau_{2}-$ semi - open $S\left(\tau_{2}\right)$-locally finite refinement $\left\{V_{\alpha}^{\prime}: \alpha \in \Delta\right\}$. Hence $\left\{U_{\beta_{0}}\right\} \cup\left\{V_{\alpha}^{\prime}: \alpha \in \Delta\right\}$ is a $\tau_{1} \tau_{2}-$ semi-open $p_{1}$-locally finite refinement of $\tilde{U}$.

Case 2: If $\cup\left\{V_{\alpha}: \alpha \in \Delta\right\} \neq X$, then $F=X-\cup\left\{V_{\alpha}: \alpha \in \Delta\right\}$ is a $\tau_{2}-$ semi - closed proper subset of $X$, and $F \subseteq \cup\left\{U_{\beta}: \beta \in \Gamma\right\}$. Hence there is a $S\left(\tau_{1}\right)$-locally finite $\tau_{1}-$ semi - open refinement of $\left\{U_{\beta}: \beta \in \Gamma\right\}$, say $\left\{U_{\beta}^{\prime}: \beta \in \Gamma\right\}$. If $\cup\left\{U_{\beta}^{\prime}: \beta \in \Gamma\right\}=X$ there is nothing to prove. But if $\cup\left\{U_{\beta}^{\prime}: \beta \in \Gamma\right\} \neq X$ then $X-\cup\left\{U_{\beta}^{\prime}: \beta \in \Gamma\right\}$ is a $\tau_{1}-$ semi - closed proper subset contained in $\cup\left\{V_{\alpha}: \alpha \in \Delta\right\}$, so there is a $\tau_{2}$-locally finite $\tau_{2}-$ semi - open refinement $\left\{V_{\alpha}^{\prime}: \alpha \in \Delta\right\}$ of $\left\{V_{\alpha}: \alpha \in \Delta\right\}$. Therefore $\left\{U_{\beta}^{\prime}: \beta \in \Gamma\right\} \cup\left\{V_{\alpha}^{\prime}: \alpha \in \Delta\right\}^{\alpha}$ is a $\tau_{1} \tau_{2}-$ semi - open $p_{1}$-locally finite refinement of $\tilde{U}$. Hence the result.

Similarly, we can prove the following theorem:

Theorem 2.11. For the bitopological space $\left(X, \tau_{1}, \tau_{2}\right)$ the following are equivalent:

$i)\left(X, \tau_{1}, \tau_{2}\right)$ is $p_{2}-$ semi-paracompact.

ii) If $F$ is any $\tau_{i}-$ semi-closed proper subset of $X$ then any $\tau_{j}-$ semi-open cover of $F$ has a $\tau_{j}$-semi - open $S\left(\tau_{i}\right)$-locally finite ( in $X$ ) refinement, $i \neq j ; i, j=1,2$.

Theorem 2.12. For the bitopological space $\left(X, \tau_{1}, \tau_{2}\right)$ the following are equivalent:

i) $\left(X, \tau_{1}, \tau_{2}\right)$ is $p_{1}-$ semi-paracompact and $p_{2}-$ semi-paracompact.

ii) Every $p$-semi-open cover of $X$ has a $S\left(\tau_{1}\right)$-locally finite $\tau_{1} \tau_{2}-$ semiopen refinement and every $p-$ semi - open cover of $X$ has a $S\left(\tau_{2}\right)$-locally finite $\tau_{1} \tau_{2}-$ semi - open refinement.

Proof. $(i) \longrightarrow(i i)$. Let $\tilde{U}$ be a $p-$ semi-open cover of $X$. Then there exist $U \in \tilde{U} \cap S\left(\tau_{1}\right)$ and $V \in \tilde{U} \cap S\left(\tau_{2}\right)$ such that $U \neq \phi$ and $V \neq \phi$. Since $\left(X, \tau_{1}, \tau_{2}\right)$ is $p_{2}-$ semi-paracompact, there exists a $p_{2}$-locally finite $\tau_{1} \tau_{2}-$ semi - open refinement $\tilde{N}$ of $\tilde{U}$. Since $\left(X, \tau_{1}, \tau_{2}\right)$ is $p_{1}-$ semi-paracompact, the $p$-semi-open cover $\tilde{N}$ ( add $U$ to the cover $\tilde{N}$ if $\tilde{N} \cap S\left(\tau_{1}\right)=\phi$ and add $V$ to the cover $\tilde{N}$ if $\left.\tilde{N} \cap S\left(\tau_{2}\right)=\phi\right)$ has a $p_{1}$-locally finite $\tau_{1} \tau_{2}-$ semi-open refinement $\tilde{O}$. Observe that $\left(\tilde{N} \cap S\left(\tau_{1}\right)\right) \cup\left(\tilde{O} \cap S\left(\tau_{2}\right)\right)$ is a $S\left(\tau_{2}\right)$-locally finite $\tau_{1} \tau_{2}-$ semi - open refinement ( replace $\tilde{N} \cap S\left(\tau_{1}\right)$ by $\{U\}$ if $\tilde{N} \cap S\left(\tau_{1}\right)=\phi$ ) of $\tilde{U}$ and $\left(\tilde{N} \cap S\left(\tau_{2}\right)\right) \cup\left(\tilde{O} \cap S\left(\tau_{1}\right)\right)$ is a $S\left(\tau_{1}\right)$-locally finite $\tau_{1} \tau_{2}-$ semi-open refinement ( replace $\tilde{N} \cap S\left(\tau_{2}\right)$ by $\{V\}$ if $\tilde{N} \cap S\left(\tau_{2}\right)=\phi$ ) of $\tilde{U}$.

By a similar technique used above we can prove that $(i i) \longrightarrow(i)$. 
Definition 2.13. In a bitopological space $\left(X, \tau_{1}, \tau_{2}\right), S\left(\tau_{1}\right)$ is said to be semi-regular with respect to $S\left(\tau_{2}\right)$ if, for each point $x$ in $X$ and each $\tau_{1}-$ semi - closed set $F$ such that $x \notin F$, there are a $\tau_{1}-$ semi-open set $U$ and a $\tau_{2}-$ semi - open set $V$ such that $x \in U, F \subseteq V$ and $U \cap V=\phi$.

$\left(X, \tau_{1}, \tau_{2}\right)$ is $p-s e m i$-regular if $S\left(\tau_{1}\right)$ is semi-regular with respect to $S\left(\tau_{2}\right)$ and vice versa.

Definition 2.14. ([8]). A bitopological space $\left(X, \tau_{1}, \tau_{2}\right)$ is said to be $p-s e m i$-Lindelöf if each $p-s e m i-$ open cover of $X$ has a countable subcover.

Definition 2.15. ([8]). A subset $A$ of a bitopological space $\left(X, \tau_{1}, \tau_{2}\right)$ is said to be $p-$ semi-Lindelöf relative to $\left(X, \tau_{1}, \tau_{2}\right)$ if every $p-$ semi - open cover of $A$, by elements in $S\left(\tau_{1}\right) \cup S\left(\tau_{2}\right)$, has a countable subcover.

Definition 2.16. Let $\left(X, \tau_{1}, \tau_{2}\right)$ be a bitopological space. A subset $D$ of $X$ is called $p-s e m i$-dense if $s C l_{1} D=s C l_{2} D=X$ and for every $p-s e m i-o p e n$ ( in X ) $p_{2}$-locally finite ( in $X$ ) countable cover $\tilde{U}$ of $D$ we have

$s C l_{1} \cup\left\{U: U \in \tilde{U} \cap S\left(\tau_{1}\right)\right\} \subseteq s C l_{2} \cup\left\{U: U \in \tilde{U} \cap S\left(\tau_{1}\right)\right\}$ or $s C l_{2} \cup\left\{U: U \in \tilde{U} \cap S\left(\tau_{2}\right)\right\} \subseteq s C l_{1} \cup\left\{U: U \in \tilde{U} \cap S\left(\tau_{2}\right)\right\}$.

Theorem 2.17. Let $\left(X, \tau_{1}, \tau_{2}\right)$ be a $p_{2}-$ semi-paracompact $p$-semi-regular space having a $p-s e m i-$ dense subset $A$ where $A$ is $p-s e m i$-Lindelöf relative to $X$. Then $X$ is $p-s e m i-$ Lindelöf.

Proof. Let $\tilde{N}$ be a $p-$ semi-open cover of $X$. Then there exist at least two non-empty sets $V_{0}, U_{0} \in \tilde{N}$ such that $V_{0} \in S\left(\tau_{1}\right)$ and $U_{0} \in S\left(\tau_{2}\right)$. Since $\left(X, \tau_{1}, \tau_{2}\right)$ is $p-s e m i-$ regular and $p_{2}-$ semi-paracompact, $\tilde{N}$ has a $p_{2}$-locally finite $\tau_{1} \tau_{2}-$ semi-open refinement $\tilde{U}$ such that for each $U \in \tilde{U} \cap S\left(\tau_{1}\right), s C l_{2} U$ is contained in some $V \in \tilde{N} \cap S\left(\tau_{1}\right)$ and for each $U \in \tilde{U} \cap S\left(\tau_{2}\right), s C l_{1} U$ is contained in some $V \in \tilde{N} \cap S\left(\tau_{2}\right)$. Now $\tilde{U}$ is $p-$ semi-open cover of $A$ by semi - open sets in $S\left(\tau_{1}\right) \cup S\left(\tau_{2}\right)$ ( add $V_{0}$ to $\tilde{U}$ if $\tilde{U} \cap S\left(\tau_{1}\right)=\phi$ and add $U_{0}$ to $\tilde{U}$ if $\left.\tilde{U} \cap S\left(\tau_{2}\right)=\phi\right)$. Since $A$ is $p-$ semi-Lindelöf relative to $X$, there exists a countable subcover $\tilde{U}^{\prime}$ of $\tilde{U}$ which covers $A$. We may assume that $\tilde{U}^{\prime}$ is $p$-semi-open. But $A$ is $p-s e m i$-dense in $X$, so we have two cases to consider:

Case 1. $s C l_{1} \cup\left\{U: U \in \tilde{U}^{\prime} \cap S\left(\tau_{1}\right)\right\} \subseteq s C l_{2} \cup\left\{U: U \in \tilde{U}^{\prime} \cap S\left(\tau_{1}\right)\right\}:$ In this case we have:

$$
\begin{aligned}
& X=s C l_{1} A \\
& \subseteq s C l_{1} \cup\left\{U: U \in \tilde{U}^{\prime}\right\} \\
& =\left[s C l_{1} \cup\left\{U: U \in \tilde{U}^{\prime} \cap S\left(\tau_{1}\right)\right\}\right] \cup\left[s C l_{1} \cup\left\{U: U \in \tilde{U}^{\prime} \cap S\left(\tau_{2}\right)\right\}\right] \\
& \subseteq\left[s C l_{2} \cup\left\{U: U \in \tilde{U}^{\prime} \cap S\left(\tau_{1}\right)\right\}\right] \cup\left[s C l_{1} \cup\left\{U: U \in \tilde{U}^{\prime} \cap S\left(\tau_{2}\right)\right\}\right]
\end{aligned}
$$




$$
=\left[\cup\left\{s C l_{2} U: U \in \tilde{U}^{\prime} \cap S\left(\tau_{1}\right)\right\}\right] \cup\left[\cup\left\{s C l_{1} U: U \in \tilde{U}^{\prime} \cap S\left(\tau_{2}\right)\right\}\right] .
$$

Case 2. $s C l_{2} \cup\left\{U: U \in \tilde{U}^{\prime} \cap S\left(\tau_{2}\right)\right\} \subseteq s C l_{1} \cup\left\{U: U \in \tilde{U}^{\prime} \cap S\left(\tau_{2}\right)\right\}:$ In this case we have :

$$
\begin{aligned}
& X=s C l_{2} A \\
& \subseteq s C l_{2} \cup\left\{U: U \in \tilde{U}^{\prime}\right\} \\
& =\left[s C l_{2} \cup\left\{U: U \in \tilde{U}^{\prime} \cap S\left(\tau_{1}\right)\right\}\right] \cup\left[s C l_{2} \cup\left\{U: U \in \tilde{U}^{\prime} \cap S\left(\tau_{2}\right)\right\}\right] \\
& \subseteq\left[s C l_{2} \cup\left\{U: U \in \tilde{U}^{\prime} \cap S\left(\tau_{1}\right)\right\}\right] \cup\left[s C l_{1} \cup\left\{U: U \in \tilde{U}^{\prime} \cap S\left(\tau_{2}\right)\right\}\right] \\
& =\left[\cup\left\{s C l_{2} U: U \in \tilde{U}^{\prime} \cap S\left(\tau_{1}\right)\right\}\right] \cup\left[\cup\left\{s C l_{1} U: U \in \tilde{U}^{\prime} \cap S\left(\tau_{2}\right)\right\}\right] .
\end{aligned}
$$

Now in each case, we get

$X \subseteq\left[\cup\left\{s C l_{2} U: U \in \tilde{U}^{\prime} \cap S\left(\tau_{1}\right)\right\}\right] \cup\left[\cup\left\{s C l_{1} U: U \in \tilde{U}^{\prime} \cap S\left(\tau_{2}\right)\right\}\right]$.

For each $U \in \tilde{U}^{\prime} \cap S\left(\tau_{i}\right)$ choose one element $V_{U} \in \tilde{N}$ such that $s C l_{j} U \subseteq V_{U}$ $(i \neq j ; i, j=1,2)$. Then $\left\{V_{U}: U \in \tilde{U}^{\prime}\right\}$ is a countable subcover of $\tilde{N}$ for $X$. Hence $X$ is $p-s e m i-$ Lindelöf.

\section{References}

[1] S. G. Crossley and S. K. Hildebrand, Semi-closure, Texas J. Sci., 22 (1971), $99-112$.

[2] - Semi-topological properties, Fun. Math., 74 (1972), 233 - 254.

[3] Ch. Dorset, Semi compactness, semi separation axioms, and product spaces, Bull. Malaysian Math. Soc., 4(2) (1981), 21 - 28.

[4] - Semi convergence and semi compactness, Indian J. Mech. Math., 19(1) (1982), $11-17$.

[5] F. Hanna and Ch. Dorset, Semi compactness, Q and A in General Topology, 2 (1984), $38-47$.

[6] J. C. Kelly, Bitopological spaces, Proc. London Math. Soc., 13 (1963), 71 $-89$.

[7] N. Levine, Semi-open sets and semi-continuity in topological spaces, Amer. math. Monthly, 70 (1963), 36 - 41.

[8] Jamal M. Mustafa, Semi compactness and semi Lidelöfness in bitopological spaces, Abhath Al-Yarmouk: "Basic sci \& Eng.", 13(2)(2004), 309 - 314.

Received: March 8, 2007 